\title{
Patients could share virtual medical appointments for better access to telemedicine
}

\author{
Shared medical appointments, whereby patients with similar medical conditions consult their medical practitioner \\ together, alleviate pressure on the health system and provide an instant support network for the patient. Why not \\ make them virtual?
}

\section{Kamalini Ramdas and Soumya Swaminathan}

\begin{abstract}
$\mathrm{n}$ the USA, shared medical appointments (SMAs) have been offered successfully for over two decades for many conditions ${ }^{1}$.
\end{abstract} Providers offering SMAs have reported improved productivity, expanded access, shorter waits and better medical outcomes - for example, improved control of blood sugar and fewer visits to the emergency department for people with diabetes, and fewer preterm births ${ }^{2}$.

In these grouped appointments, because clinicians need to share advice that is common to the patient group only once, they have more time to delve into more complicated topics and to reflect on each patient's individual specificities. SMAs also enable peer support and peer learning.

The Cleveland Clinic in the USA has been offering SMAs in every department and was seeing several thousand patients a month in SMAs before the COVID-19 pandemic. SMAs have also been used in Australia, including use for aboriginal populations, in the UK's National Health Service, and in some low- and middle-income countries ${ }^{3}$. In India, the Aravind Eye Hospital, which sees 3.5 million patient visits a year, has successfully trialed in-person SMAs for patients with glaucoma ${ }^{4,5}$.

At a time when many countries have increasingly been leveraging digital technologies, virtual SMAs (VSMAs) can greatly increase telehealth capacity by increasing the capacity and effectiveness of existing telehealth platforms and by mitigating the mismatch between care supply and care demand ${ }^{6}$.

VSMAs can also expand access to underserved areas. Private telehealth providers have shown little interest in those in poor rural communities who cannot afford high fees for private consultation and may lack internet access, especially in low- and middle-income countries. In India, for instance, the rural population - an estimated $65 \%$ of the overall population still has meagre internet access. Offering telehealth to these underserved populations requires building a fixed-base telemedicine network through which patients can remotely access care ${ }^{7}$, which can potentially be costly. Grouping remote appointments could reduce access fees due to the lower clinician time needed per patient in grouped appointments and could thus make remote appointments more accessible to underprivileged populations.

Moreover, instead of being scattered across a variety of care centers, patients accessing a VSMA could be grouped by local community, which would enable them to share valuable local knowledge, such as where to find locally available healthy foods or health amenities, and to form supportive bonds. These interactions would increase patient capacity for self-care, the likelihood of compliance with medications and other instructions, and community engagement.

Many of the technological building blocks for transition to VSMAs are already in place. For example, most telehealth platforms already allow multiple generalist doctors to connect securely at once to specialist doctors. However, some hurdles remain to be overcome for VSMAs to be effective.

Mobile-phone penetration remains a challenge, and governments will need investment in IT infrastructure, as well as in modifying the way primary-care clinics operate. One example of an innovative approach to care provision is the Indian government's eSanjeevani platform ${ }^{8}$, which connects smartphone-equipped community health officers in 3,000 rural health and wellness centers (HWCs) to general practitioners and specialist doctors through a hub-and-spoke model (Fig. 1). The platform also offers direct doctor-to-patient consultations for patients who have internet access. Over 12,000 practitioners have already been brought on board and 0.4 million consultations have been delivered. If VSMAs are offered at rural health and wellness centers, auxiliary providers from nearby towns - for example, nutritionists, physiotherapists or pharmacists - may find it worthwhile to travel to these remote locations and may worry less about 'no-show' patients.

Switching to VSMAs also requires rigorous trialing combined with mentoring, training and behavior change for both patients and providers. Adoption of SMAs has been slow ${ }^{9}$, limited by inadequate scientific evidence, especially on how SMAs affect patients' behaviors or experiences ${ }^{10}$. Many providers and patients worry that lack of privacy can compromise patients' experiences. Rigorous trialing of SMAs - and VSMAs - through large-scale prospective trials will build scientific evidence on how these care models affect such experiences.

But quickly transitioning to a radically different care delivery model is an achievable goal. In the COVID-19 pandemic, most in-person SMAs at the Cleveland Clinic have transitioned to VSMAs, and hundreds of physicians and staff have been trained through internal webinars. VSMAs have proven as effective as SMAs for weight loss and type 2 diabetes, in small-sample, retrospective studies ${ }^{11,12}$. Training platforms such as Project ECHO, an online platform that transfers knowledge from specialists at urban academic care centers to rural clinicians in many countries $^{13}$, can accelerate the adoption of VSMAs and guide implementers on how to gather data to scientifically validate this care model.

Thinking of new ways to improve healthcare delivery is particularly important at a time when the COVID-19 pandemic has had huge impact on health-service delivery worldwide. A pulse survey conducted by the World Health Organization across 120 countries showed that essential health services were disrupted in over $80 \%$ of the countries. Low-income countries have been disproportionately impacted, with $45 \%$ of essential services disrupted, while in high-income countries, this has been less pronounced, at $4 \%$. Countries in the Eastern Mediterranean Region have been most 


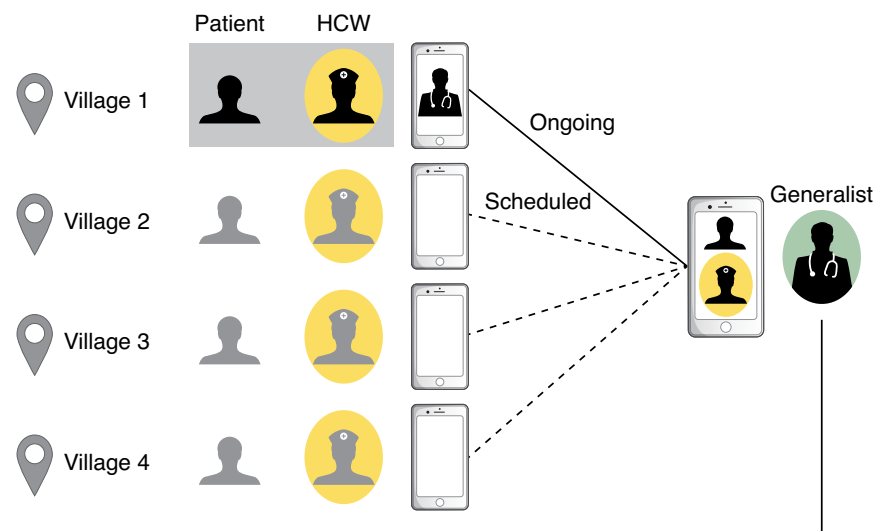

b

VSMA with patients from many villages

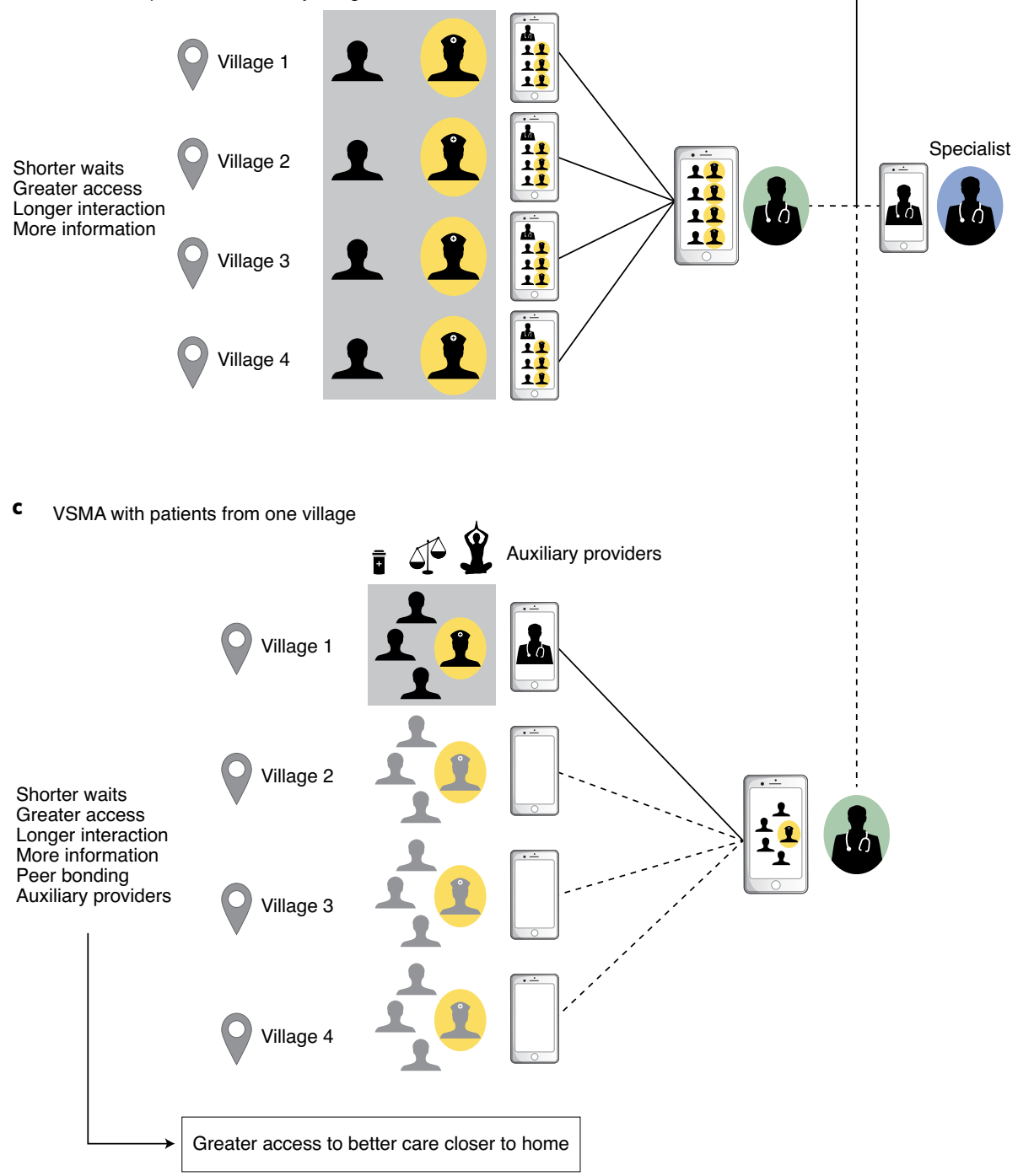

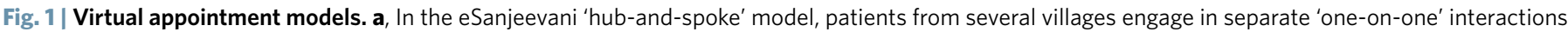
(1-1s) with physicians, aided by healthcare workers (HCWs), at their village health and wellness centers (HWCs). b, In a VMSA that spans several villages, patients engage in simultaneous remote interactions with physicians and with one another, aided by HCWs. c, In a VMSA at one village, patients interact in

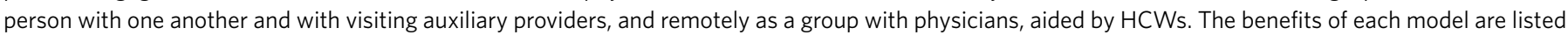
at left. Solid lines indicate appointments that are in process; dashed lines indicate scheduled appointments. 
affected, followed by those in the African and Southeast Asian regions ${ }^{14}$. This will probably result in increases in mortality and morbidity due to non-COVID-19 diseases and a setback for the health of populations worldwide.

The World Health Organization's Global Strategy on Digital Health ${ }^{15}$, which was adopted by the World Health Assembly in November 2020, is a call to action and provides a roadmap for countries to expand digital health services rapidly. The COVID-19 pandemic has provided an opportunity to leapfrog into a reality of 'Health for All' through the use of new digital tools, but this needs to be accompanied by innovation in systems thinking and a willingness to learn and adapt quickly.
Kamalini Ramdas $\mathbb{D}^{1,3 凶}$ and

Soumya Swaminathan ${ }^{2,3}$

${ }^{1}$ Management Science and Operations, London Business School, London, UK. ${ }^{2}$ World Health

Organization, Geneva, Switzerland. ${ }^{3}$ These authors contributed equally: Kamalini Ramdas, Soumya

Swaminathan.

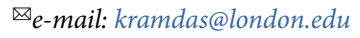

Published online: 13 January 2021

https://doi.org/10.1038/s41591-020-01187-4

References

1. Noffsinger, E.B. Running Group Visits in your Practice (Springer Science \& Business Media, 2009).

2. Jones, T. et al. Future Healthc. J. 6, 8 (2019).

3. Sharma, J., O'Connor, M. \& Rima Jolivet, R. Reprod. Health 15 , 38 (2018).

4. Venkatesh, R., Kavitha, S., Sonmez, N., Buell, R. \& Ramdas, K. Invest. Ophthalmol. Vis. Sci. 58, 2116 (2017).

5. Buell, R., Ramdas, K. \& Sonmez, N. SSRN https://papers.ssrn. com/sol3/papers.cfm?abstract_id $=3646420$ (2020).
6. Ramdas, K., Ahmed, F. \& Darzi, A. Lancet Digit. Health 2, e288e289 (2020).

7. Delana, K., Deo, S., Ramdas, K., Babu, G. \& Ravilla, T. SSRN https://papers.ssrn.com/sol3/papers.cfm?abstract_id=3505318 (2020).

8. Agrawal, A. Science 369, 1050-1052 (2020).

9. Ramdas, K. \& Darzi, A. N. Engl. J. Med. 376, 1105-1107 (2017).

10. Menon, K. et al. Front. Endocrinol. 8, 263 (2017).

11. Shibuya, K., Pantalone, K. M. \& Burguera, B. Endocr. Pract. 24, 1108-1109 (2018).

12. Tokuda, L. et al. Int. J. Med. Inform. 93, 34-41 (2016).

13. Zhou, C., Crawford, A., Serhal, E., Kurdyak, P. \& Sockalingam, S. Acad. Med. 91, 1439-1461 (2016).

14. World Health Organization. https://www.who.int/publications/i/ item/WHO-2019-nCoV-EHS_continuity-survey-2020.1 (2020).

15. World Health Organization. https://www.who.int/docs/defaultsource/documents/gs4dhdaa2a9f352b0445bafbc79ca799dce4d. pdf?sfvrsn=f112ede5_68 (2020).

Acknowledgements

We thank S. Katuri for her excellent research assistance.

Competing interests

The authors declare no competing interests. 